\title{
A Multi-Layer Distributed Comprehensive On-line Monitoring System for GIS
}

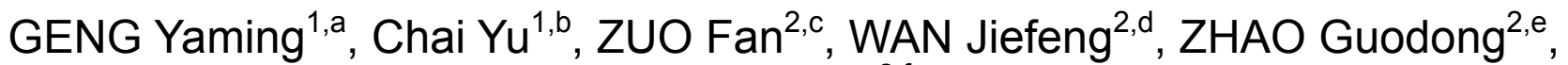 \\ ZHU Meng ${ }^{2, f}$
}

${ }^{1}$ Operating\&Maintenance Station, Taizhou Maintenance Branch, Jiangsu Electric Power Co, Taizhou 225300;

${ }^{2}$ School of Electrical Engineering, Southeast University, Nanjing 210096, China.

a6736639@qq.com, ${ }^{\mathrm{b}}$ chajy@163.com, ${ }^{\mathrm{c}}$ 850527240@qq.com, ${ }^{\mathrm{d}} 983082574 @ q q . c o m,{ }^{\mathrm{e}} 281636528 @ q$

q.com, ${ }^{f} 472881284 @ q q . c o m$

Keywords:GIS, Distributed structure, IEC61850, On-line monitoring, ARM

\begin{abstract}
Gas Insulated Switchgear (GIS), as important equipment of power system, providesprimaryguarantee for its security and stability. Therefore it is significant to monitor the operating statusand working performance of GIS. In this paper, acomprehensive online monitoring system of GISwith amulti-layer distributed structure is proposed.The design realizes the monitoring of many important parameters of GIS, including the temperature of gas, the pressure of gas, operating status, three-phase mechanical properties, the characteristics of control loop, etc. The system has lots of functions such as the collectionof data, the storage and management of data, the calculation of parametersand remote communication. Field experimentsverify that the system is accurate and effective enough to meet the requirements of online monitoring.
\end{abstract}

\section{Introduction}

GIS(Gas Insulated Switchgear) isimportant electrical equipment which is widely used intransmission, distribution and substation fields ${ }^{[1]}$.After the optimum design, the primary equipment of a substationis integrated a whole except the transformer.GIS has lots of advantages such as smalleroccupied area, higher reliability, stronger security and less maintenance work.However, Due to its feature of seal, it's more difficult to find its early failures are than traditional circuit breakers and those failures often cause more losses. To keep abreast of the equipment's operating conditionsand find potential faults,online monitoring of GIS becomes a must ${ }^{[2]}$. At present, periodic maintenance is still widely used for most electrical equipment in China. But the traditional planned maintenance costs a lot of material and financial resources and will result in thedecrease ofreliability of power supply. Therefore,condition-based maintenance is an inevitable trend of development. Condition-based maintenance has more advantages than regular maintenance ${ }^{[3]}$. Real-time dynamic online monitoring can provide earlyfaultwarning effectivelyand accurately. It can alsoprovide the basis for the diagnosis and condition-based maintenance. In this paper,characteristic of main variablesof $220 \mathrm{kV}$ GIS and corresponding monitoring methods are analyzed torealize the multi-tier distributed design of GIS online monitoring system which is based on IEC61850. The system featureshigh synthesis, excellent expan sibility and stability by monitoring multi-variables.

\section{Main variables monitoredbythe system}

In this paper, the proposed online monitoring system is used to monitor ZF40-126 GIS. Due to the sealed nature of GIS and itscomplex internal structure, parameters must be monitored to reflect both the status of GIS and the important factors which may cause the fault. With the requirements of the monitoring system and the current monitoring methods taken into consideration, theparameters includingcircuit current of SF6 circuit breakers, grounding switch status, SF6 gas temperature and pressure are monitoredin the paper ${ }^{[4]}$. Specific monitoring parameters are as follows: 
1) The circuit breaker closing state and cumulative breaking times. The cumulative breaking times can be used to determine the electrical endurance of circuit breakers.

2) The state of ground knife. During the maintenance, grounding switch must be closed unless the door of switchgear cannot be opened. It is necessary to grasp thestate of ground knife for safety.

3) The travel of three-phase breaker moving contact. By calculating the three-phase circuit breaker moving contact stroke, some valuable mechanical parameters can be gained such asthe speed of opening and closing, the times ofopening and closing, open distance, three-phase different period,etc.

4) The current signal of closing coil. Monitoring the changes ofDC coil current signal in the process of opening and closing can reflect secondary operations coil condition, operating status of solenoids and other mechanical components.

5) The current signal of charging motor. Through the current signal of the circuit breaker charging motor, the operation of electrical energy storage can be observed.

6) Mechanical vibration signal. The signal includes a number of circuit breaker operation status and internal mechanical structure information ${ }^{[5]}$.

7) The temperature and pressure signals of SF6 gas. Temperature and pressure are usually monitored by sensors and then converted into corresponding purity and density.

\section{The overall structure of the monitoring system}

Conventional monitoring systems typically use a two-layer structure-upper and lower computer. The upper and lower computer communicate throughCAN bus and RS232 serial ports ${ }^{[6]}$. This structure has many shortcomings such as the high cost of the lower computer, the lack of the capacity of sampling rate and overall treatment, and less overall practicability. The communicatemode using CAN bus and RS232 serial portleads to obvious deficiency of data transmission speed and interoperability. According to the basic requirements for GIS on-line monitoring, combined with GIS's own characteristics, in order to adapt to the development trend of intelligent substation and meet the interoperability requirements, this system uses a multi-tier distributedstructure in tree form based on IEC61850 standard.

IEC61850 standard divides the substation automation systems into substation layer, the spacer layer and process layer. This stratificationis not only logical, but also is physical. It makes both the logical interface and the physical structure of the entire system clear.Process layer mainly conductsthe functions related to the primary equipment such as sending the order of action command and the collection of the switching value and the analog quantity. It communicates with the spacer layer via Ethernet. Spacer layerusesthe data of the layer to operate the primary equipment inthat layer. Individual devices in the spacer layer may also communicate with each other via a logical interface.Substation layercommunicates withspacer layer via Ethernet based on TCP / IP, which replaces the traditional way of CAN bus and increases the speed of communication and interoperability.

The entire system consists of multiple-sensors, data receivingand communicationunit,distributed on-site monitoring and control unit, back-end server and other components. Each layer can communicate with each other rapidly through embedded Ethernet.Data receivingand communicationunit consists of ARM module and FPGA module, which is responsible formonitoring the collection of dataand remote communication. Distributed on-site monitoring and control unit, which is made up of the IPC with a touch screen, is responsible forthe monitor of human-machine interface display data, historical data query and fault warning. Back-end server is used to monitor all devices, and implement remote operation. The multi-liar distributed structure can not only give full play to each part's superiority, but also make rational use of IPC's powerful calculating and analyzing capabilityand advantages of embedded processors such as fast communication and small volume.Based on IEC61850 standards, the design meets the modularization development and features good expansibility, interoperability as well as stability. 


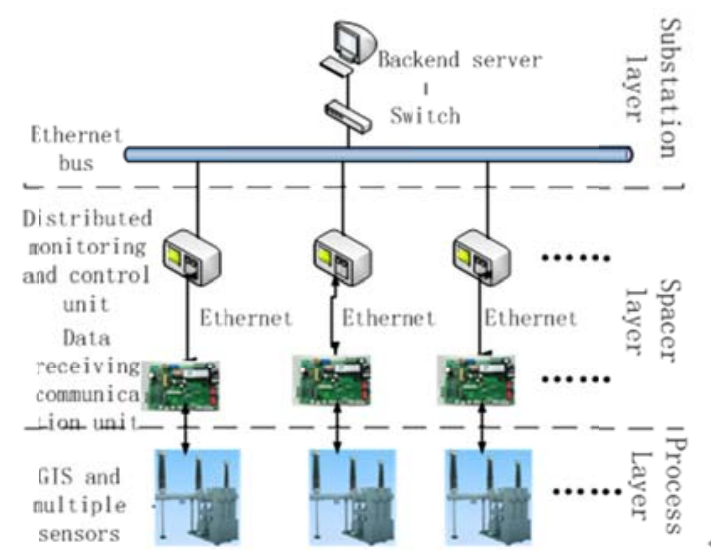

Fig.1 The monitoring system structure as a whole

\section{The hardware design of GIS online monitoring system}

As shown in Fig.1, GIS on-line monitoring device belongs to spacer layer, including the unit of data receivingand communication, the unit of distributed on-site monitoring and control, whichis an important component of the system. It is supposed to not only receive the status information ofprimary equipment and send it tobackstage server, but also communicatethe commands of serversto primary equipment.

Main structure of the system hardware design is shown in Fig. 2. SF6 sensor signal isconvertedby the RS485 / 232 directly,thenthe signal is connected to the RS232 serial of IPC. Switch signal is sent to the ARM chip on the core board via I/O board. The remaining analog signals need pretreatment through acquisition board before the core board completes the A/D conversion and sends the signals to ARM chip. Finally, ARM packages all switch signals and analog signals, and sendsthem to the IPC for processing via Ethernet.IPC can call the relevant algorithm to deal with those dataand display them on man-machine interface. By analyzing the data,IPC canwarn faults early and control operations of GIS.

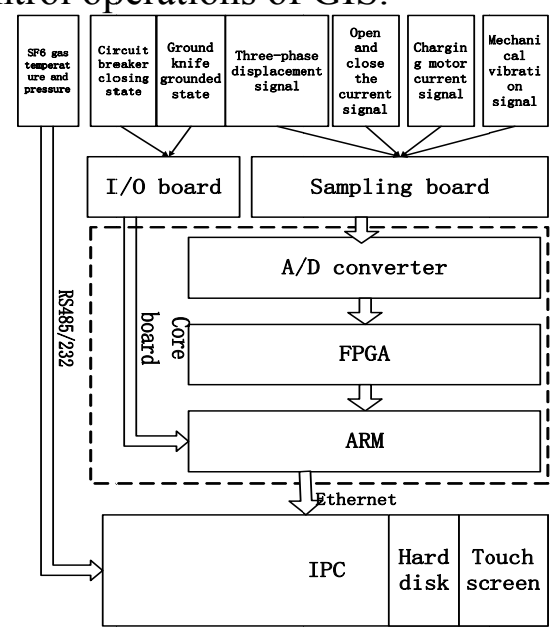

Fig.2 Hardware structure

\section{Hardware design ofsignal conditioning modules}

Signal conditioning modules consists of sampling board and I/O board. The channel outputof the $\mathrm{I} / \mathrm{O}$ board is connected to the corresponding I/O pins of the ARM chip on the core board directly.I/O channels are isolatedby optocoupler to achieve the isolation of internal and external signal of input and output. It can enhance electromagnetic compatibility and interference immunity besides protecting the internal circuit. The output channel adds monostable trigger circuit to the front end of optocoupler to filter the impulse wave of shorter pulse width at the power-on moment in cooperation with NAND gate logic circuit so that the relay malfunction caused by powering on and other interference can be avoided.Sampling board mainly acts asthe low-pass filter and pressure 
regulator ofthe analog signals. According to Shannon sampling theorem, if the signal bandwidth is less than half of the sampling frequency, these discrete sampling points could be fully representative of the original signal. If it is higher or same as the sampling frequency, itwill result in aliasing. The sampling frequency of the design is $25 \mathrm{kHz}$. Therefore, a low-pass filter is applied to filter out the signal whose frequency is higher than $12.5 \mathrm{kHz}$.Because the transition process first-order RC filter operatesslowly, a passive second-order RC filter isused.

\section{The design of core board}

There are two programmable embedded chips on the core board. The one is Cyclone 2-generation FPGA ofAlteraand the other is AT91RM9200 ARM9 seriesof Atmel. The main task of FPGA is to control the A/D conversion of the A/D chipsat the fixed sampling rate and FIFO sort of data. FPGA also sends data to ARM through bus. The main task of ARM is to receive the data from FPGA and send to IPC in the form of Ethernet packets.

The core board uses dual-core design and controls the cooperation betweenFPGA and ARM by the interrupt signal. Software flow chart is shown in Fig.4. FPGA sends configuration signal to set the $\mathrm{A} / \mathrm{D}$ chip to start $\mathrm{A} / \mathrm{D}$ conversion. When $\mathrm{ARM}$ receives an interrupt signal from the FPGA, it begins to accept signals from the A/D sampling chip and detects switch status at the same time. When ARM receives ten groups of interrupt signal, it starts to pack them and send them via Ethernet.

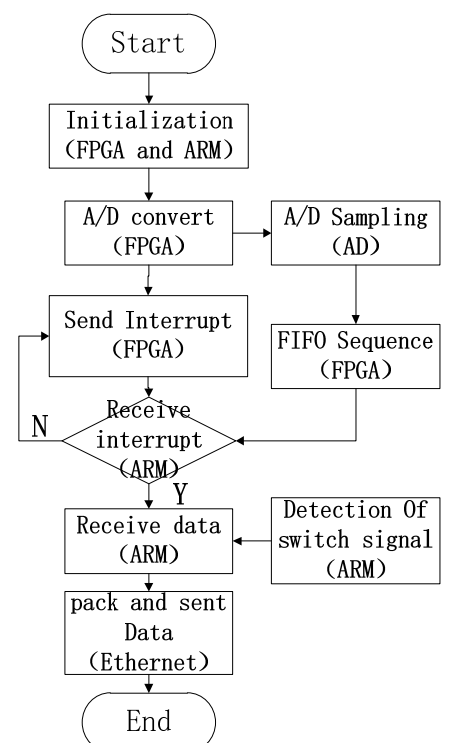

Fig.4 The flowchart of Core board software

\section{Experimental data and analysis}

The entire system is mounted on ZF40-126 GIS and the ABC three-phase displacement of SF6 circuit breaker in GIS is tested to provethe accuracy and reliability of the on-line monitoring device. IPC invokes correspondingalgorithm to eliminate noise and calculate the mechanical parameters. Results of travel data in the experimentare shown in Table1. Errors between online monitoring data and predelivery test results are shown in Table2.

Tab.1 experiment dataof GIS's mechanical parameters

\begin{tabular}{ccccc}
\hline Measurement parameters & \multicolumn{2}{l}{ Travel data of online monitoring system } & Factory data \\
\hline & A phase & B phase & C phase & Three Phase \\
Contact travel $(\mathrm{cm})$ & 12.148 & 12.252 & 11.85 & 12.000 \\
The contact trip $(\mathrm{cm})$ & 2.8457 & 3.1152 & 2.939 & 3.0000 \\
Closing sequence & & BAC & & BAC \\
Closing speed $(\mathrm{m} / \mathrm{s})$ & 2.283 & 2.331 & 2.167 & 2.200 \\
Opening sequence & & CAB & & CAB \\
Opening speed $(\mathrm{m} / \mathrm{s})$ & 4.981 & 4.952 & 5.046 & 5.000 \\
\hline
\end{tabular}


Tab.2errors of GIS on-line monitoring system

\begin{tabular}{cccc}
\hline Measurement parameters & \multicolumn{3}{c}{ Error } \\
\hline & A phase(\%) & B phase (\%) & C phase (\%) \\
Contace travel & 1.23 & 2.10 & 1.24 \\
The contact trip & 5.14 & 3.84 & 6.13 \\
Closing speed & 3.78 & 5.95 & 1.50 \\
Opening speed & 0.38 & 0.96 & 0.92 \\
\hline
\end{tabular}

The result of Tab.2 shows that the system is suitable for on-line monitoringof GIS.It also shows that testdata is close to the predelivery dataas travel, the order of switching, the speed ofswitching and other mechanical parameters are relatively accurate. Therefore the system canconduct the task of on-line monitoring successfully.

\section{Conclusion}

According with the development tendency of intelligent substation,the proposed system makes a huge change to the online-monitoring of the primary devices on the process layerof traditionalsubstation. The multi-tier distributed GIS online monitoring system can monitor various parameters, whichmeets IEC61850 standards. Its reasonable structure of the hardware layout can achieve a clear division and coordination between layers. It alsomeets the instantaneity, reliability, interoperability requirements. Obtainingonline monitoring data isthe basis for condition-based maintenance and fault diagnosis. With the development of smart grid, the on-line monitoring system proposed in this paper will have wide application.

\section{REFERENCES}

[1]Dale Rogerson. Inside Com. Redmond[M]. Washington, U.S.A: Microsoft Press, 1997.

[2] Loeb L B. Electrical Coronas. University of California Press, 1965.

[3]M • Landry etal Benefit Case studies of On-line Condition Monitoring System for HVCBISH.97 Canada 1997.

[4]Breg D, works G N. Effect of space charge on electric breakdown of sulfur hexafluoride in noun inform fields. AIEE Trans, 1958, Vol.77:p820 823.

[5]Charbkeaw N, Sunwanasri Thanapong, Bunyagul Teratam. Mechanical defect detection of SF6 high voltage circuit breaker using wavelet based vibration signal analysis[J]. Telecommunications and Information Technology, 2008, ECTICON, 2008, (2): 901-904.

[6]IEC62063, High-voltage Switchgear and Control Gear-the Use ofElectronic and Associated Technologies in Auxiliary Equipment ofSwitchgear and Control gear[S]. 